\title{
Prospecção Tecnológica da Espécie Cyperus esculentus L.: um panorama sobre a produção científica e tecnológica
}

\author{
Technological Prospection of the Species Cyperus esculentus L.: a \\ panorama about the scientific and technological production
}

\author{
Renata Correia ${ }^{1}$ \\ Reinaldo Assunção Júnior ${ }^{2}$ \\ Romulo Spósito ${ }^{3}$ \\ Cristina Pungartnik ${ }^{4}$ \\ ${ }^{1}$ Universidade Estadual do Sudoeste da Bahia, Vitória da Conquista, BA, Brasil \\ ${ }^{2}$ Engenheiro Agrônomo, Ipanema, MG, Brasil \\ ${ }^{3}$ Instituto Federal de Ciências e Tecnologia, Itapetinga, BA, Brasil \\ ${ }^{4}$ Universidade Estadual de Santa Cruz, Ilhéus, BA, Brasil
}

\begin{abstract}
Resumo
O presente trabalho teve como objetivo mapear as produções científicas e tecnológicas relacionadas à Cyperus esculentus, avaliando as potencialidades dessa espécie no cenário mundial. Foi realizada uma busca nas bases de dados de patentes do European Patent Office (EPO) e nas bases de dados de periódicos Web of Science, Science Direct e Scielo. A busca encontrou 698 patentes e 118 artigos. O ano de 2011 apresentou o maior número de patentes depositadas e a China aparece como o maior depositante. Os resultados da Classificação Internacional de Patentes estão restritos à Seção A, atribuída a necessidades humanas, em destaque para a Subseção A23L, associada a produtos alimentícios. Em relação às áreas de pesquisa, houve destaque para a área de Agricultura. Os resultados também evidenciaram a potencialidade tecnológica e científica da espécie para a indústria farmacêutica, pois as publicações e patentes analisadas relatam atividade terapêuticas, bem como a presença de compostos bioativos.
\end{abstract}

Palavras-chave: Cyperus esculentus L. Prospecção Tecnológica. Patentes.

\begin{abstract}
The present work had as objective to map the scientific and technological productions related to Cyperusesculentus, evaluating the potential of this species in the world scenario. A search was conducted in the patent databases of the European Patent Office (EPO), and in the periodical data base, Web of Science, Science Direct, Scielo. The search resulted in 698 patents and 118 articles. The year of 2011 presented the biggest number of deposited patents. The China appears as the biggest depositor. The results of the International Patent Classification are restricted to Section A, attributed to human needs, in particular the subsection A23L associated with food products. In relation to the research areas, the area of Agriculture was highlighted. The results also showed the technological and scientific potential of the species for the pharmaceutical industry, since the publications and patents analyzed report therapeutic activity, as well as the presence of bioactive compounds.
\end{abstract}

Keywords: Cyperus esculentus L. Technological Prospection. Patents.

Área Tecnológica: Alimentos. Produtos Naturais. Alimentos. Saúde. 


\section{Introdução}

A espécie Cyperus esculentus L. está entre os representantes da família Cyperaceae, que possui cerca de 3.000 espécies, dentre as quais 2.020 foram identificadas como plantas infestantes (MELLO, 2003). É conhecida pelos nomes vulgares de Tiririca, Tiririca-amarela, Tiririca-mansa, Juncinho, Junça e Chufa. Provavelmente, é originária do Mediterrâneo e sudoeste da Ásia e, atualmente, é amplamente distribuída em regiões tropicais, subtropicais e temperadas em todo o mundo (HOLM et al., 1991; FOLLAK et al., 2016), sendo descrita como uma pequena erva graminóide perene, ereta, com caule de seção triangular, sem ramificação ou nós, glabro, com 20 a $90 \mathrm{~cm}$ de altura (LORENZI, 2008).

Cyperus esculentus trata-se de uma planta invasora e é considerada uma das ervas daninhas mais agressivas em todo mundo, devido a sua ampla distribuição e métodos reprodutivos (ARIAS-PEREZ; ESPINOSA-CAMILO; DOMINGUEZ-MONGE, 2017; GUSMAN-KANTUN; ESPINOSA-CAMILO; CAMPOS-FIGUEIROA, 2017). Sua reprodução quase exclusivamente vegetativa e a liberação de compostos fenólicos com efeitos alelopáticos confere à espécie uma capacidade competitiva que torna difícil o seu controle, acarretando impactos negativos a culturas agrícolas (OLIVEIRA JR. et al., 2011).

O sistema subterrâneo da planta é caracterizado pela presença de tubérculos nas extremidades dos rizomas (KISSMANN, 1997). Esses tubérculos são ricos em amido (ADEBOWALE; KAREEM; SOBUKOLA, 2017), proteínas e gorduras (ADEGUNWA; ADELEKAN; ADEBOWALE, 2017) e, popularmente, têm sido utilizados para preparação de uma bebida refrescante chamada "Horchata De Chufas" (OLADELE; AINA, 2007). Além disso, Cyperus esculentus tem sido utilizada pela medicina popular (MATOS et al., 2008; ARAFAT et al., 2009) para tratamento de flatulência, de indigestão, de diarreia e de sede excessiva (ADEJUYITAN, 2011).

Nesse sentido, o mapeamento de informações sobre o potencial biológico dessa espécie pode auxiliar na determinação de estratégias de pesquisas e identificação de novas propriedades e aplicação tecnológica. De acordo com Simões et al., (2012) trabalhos de prospecção são relevantes, pois permitem conhecer tendências de crescimento de uma determinada área de conhecimento ou produto de interesse. Para Lima et al. (2015), o levantamento de informações sobre publicações e patentes prevê a possibilidade de avanços científicos e pode influenciar na determinação de trajetórias tecnológicas que garantam a competitividade e sobrevivência das instituições de pesquisa. Sendo assim, pode direcionar o desenvolvimento de novas tecnologias e contribuir para o desenvolvimento científico-tecnológico e econômico.

Portanto, este trabalho tem o objetivo de analisar publicações e registros de depósitos de patentes relacionados à espécie Cyperus esculentus a fim de traçar o perfil das produções científicas e avaliar a geração de produtos e processos desenvolvidos a partir dessa planta.

\section{Metodologia}

Para a realização da prospecção tecnológica, foi feita uma busca de patentes na base de dados europeia Espacenet (Europen Patent Office - EPO). Esse banco de dados se refere à coleção completa de pedidos de patentes em mais de 80 países, incluindo, por exemplo, os pedidos 
de patentes nacionais, Instituto Nacional de Propriedade Industrial (INPI), norte-americanos, United States Patent and Trademark Office (USPTO), e via Patent Cooperation Treaty (PCT). Já para o levantamento das publicações científicas foram utilizadas as bases de dados Web of Science, Science Direct, Scielo.

Para estabelecer a estratégia de busca de documentos referentes à Cyperus esculentus determinaram-se áreas de interesse relacionadas com possíveis aplicações tecnológicas da espécie. Também foram selecionados códigos do IPC (Classificação Internacional de Patentes) sendo eles A61K (Preparações para uso médico e dentário), A61K36/00 (Preparações medicinais de constituição indeterminada contendo material de algas, líquenes, fungos ou plantas, ou seus derivados) e A61K36/8905 (Preparações medicinais relacionadas ao gênero Cyperus) (Fluxograma 1).

Fluxograma 1 - Estratégia utilizada para pesquisa de documentos relacionados à espécie Cyperus esculentus. CE (Cyperus esculentus), CEP (Cyperus esculentus and pharmacoly) CEC (Cypeus esculentus and chemistry), $\mathrm{CEH}$ (Cyperus esculentus and health), CEF (Cyperus esculentus and food), CEA (Cyperus esculentus and agriculture), YN (Yellow nutsedge)

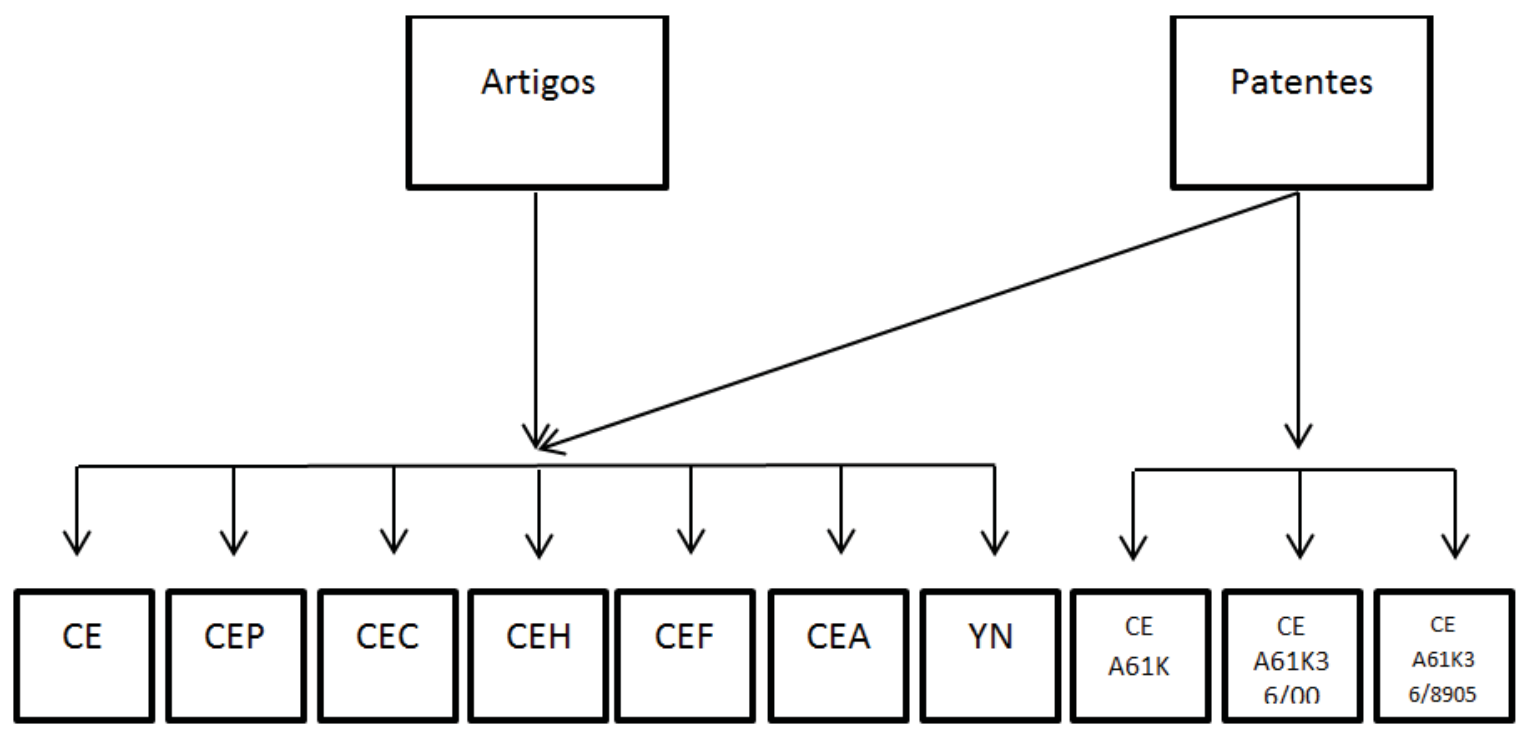

Fonte: Elaborado pelos autores deste artigo

Foram selecionados apenas os documentos que se referiam à espécie em estudo, $e$ as informações foram organizadas considerando-se ano de publicação, número de documentos, países e as principais áreas de estudo e aplicação tecnológica referentes à Cyperus esculentus.

\section{Resultados e Discussão}

Os documentos relacionados à espécie Cyperus esculentus, nos últimos 20 anos, totalizaram uma produção de 698 artigos e 118 patentes (Gráfico 1), sendo 2011 o ano com o maior número de patentes e 2016 aquele com mais publicações. Os resultados evidenciaram um aumento gradual de documentos, demonstrando a possibilidade de utilização da planta em diferentes áreas, bem como seu potencial para a geração de tecnologias. 
Gráfico 1 - Números de documentos relacionados à espécie Cyperus esculentus no período entre 1997 e 2017

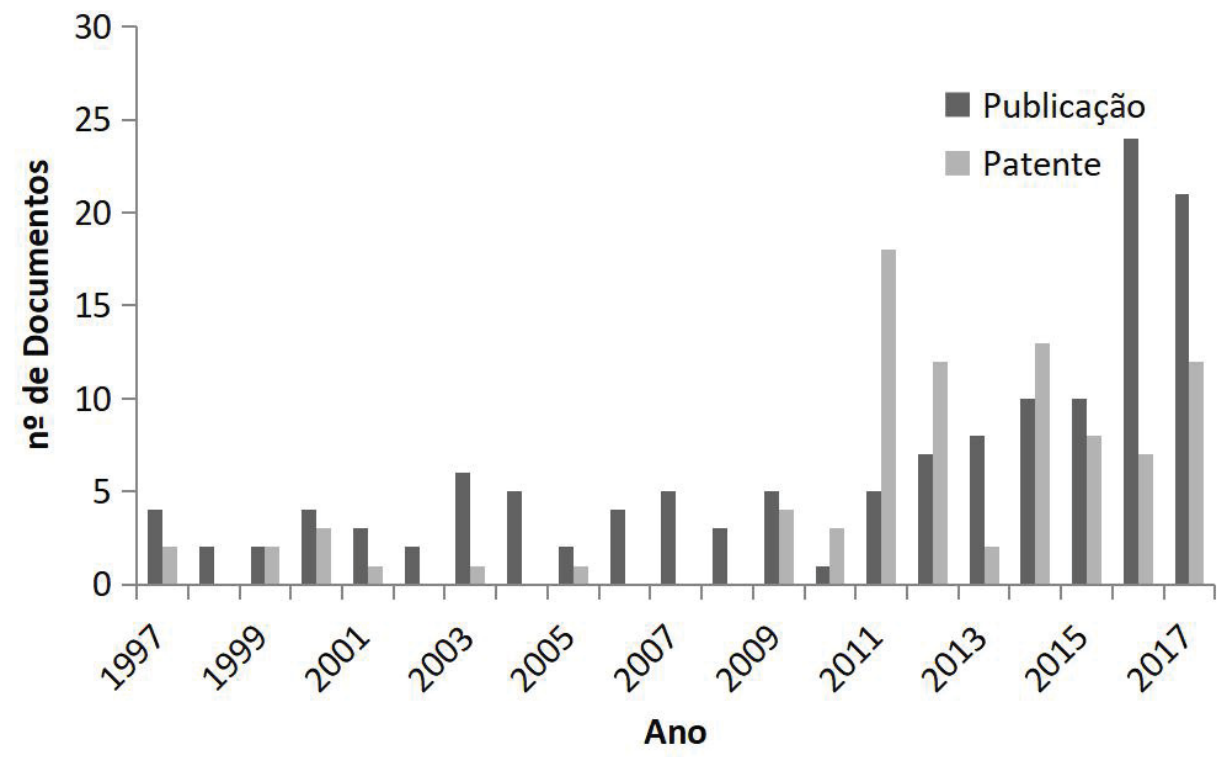

Fonte: Elaborado pelos autores deste artigo (2018)

Em relação às áreas de pesquisas científicas para o termo Cyperus esculentus L. (Gráfico 2), foi observado o maior percentual de publicações para a de área de Agricultura (65,19\%), que está associada, de modo geral, a estratégias de manejo e controle da planta (JOHNSON; WAY; BEALE, 2015; MHLANGA et al., 2016) e à avaliação do impacto da espécie sobre outras culturas (MEYERS; SHANKLE, 2017). Por se tratar de uma espécie invasora de grande impacto econômico (FOLLAK et al., 2016), justifica-se a expressiva quantidade de publicações com ênfase agronômica.

Gráfico 2 - Percentual de publicações por área de pesquisa com o Cyperus esculentus L.

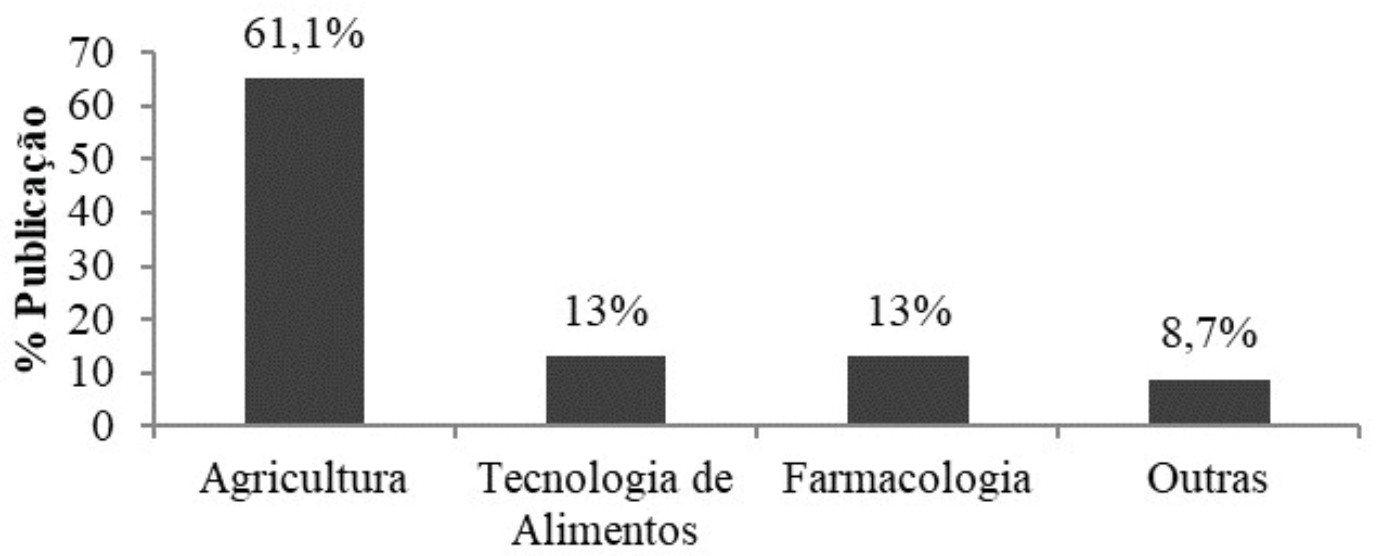

\section{Áreas}

Fonte: Elaborado pelos autores deste artigo (2018)

As pesquisas na área de Tecnologias de Alimentos (13,04\%) englobam estudos sobre o potencial nutricional dos tubérculos, evidenciando a alta concentração de lipídios e carboidratos (CODINA-TORRELLA; GUAMIS; FERRAGUT, 2017; BADO et al., 2015) e sua utilização para 
a produção de leite, farinha e bebidas (OKYERE; ODAMTTEN, 2014; AGUILAR et al., 2015; RUBERT; MONFORTE; HURKOVA, 2017), bem como suplemento nutricional (ADEBOWALE; KAREEM; SOBUKOLA, 2017; ADEGUNWA; ADELEKAN; ADEBOWALE, 2017; RUBERT; MONFORTE; HURKOVA, 2017). Os resultados revelam que os tubérculos possuem composição química e propriedades funcionais que atribuem alto valor nutritivo à planta, sendo de grande interesse para a qualidade nutricional e processamento de alimentos. Também foi verificado que pesquisas que utilizam os tubérculos da planta têm destacado o seu alto potencial oleaginoso e sua utilização para a produção de biodiesel (WANG et al., 2013).

Já os estudos farmacológicos $(13,04 \%)$ abordam sobre compostos bioativos, perfil fitoquímico e propriedades biológicas com a finalidade de obter bioprodutos. As principais potencialidades farmacológicas encontradas nas publicações apontam para a atividade antibacteriana dos tubérculos e frutos de Cyperus esculentus (PRAKASH; RAGAVAN, 2009; SEUKEP et al., 2013), a identificação de flavonoides com ação contra as bactérias Bacilus subtilis e Escherichia coli (JING; WANG, 2016) e atividade antioxidante (ADEWUYI; ODERINDE; AYODELE, 2012; JING et al., 2013; HAMDY et al., 2017). Também foi verificada a utilização da planta no tratamento de infertilidade (ALLOUH et al., 2015; SAGANUWAN, 2015). Nesse sentido, as pesquisas revelam que a espécie apresenta propriedades terapêuticas importantes, com aplicação em diferentes áreas. Sobre o perfil fitoquímico, os trabalhos descrevem a presença de flavonoides, taninos e esteroides (ADENIYI; ADEONIPEKUN; OMOTAYO, 2014).

A análise das áreas de aplicação das tecnologias patenteadas, em relação à Classificação Internacional de Patentes (IPC), demonstrou que a maioria dos depósitos está atribuída à Necessidades Humanas, com 93,41\% dos registros, que abrangem produtos e processos voltados para a área de agricultura, saúde e alimentos; e ainda, artigos de uso pessoal e doméstico (Gráfico 3).

Gráfico 3 - Percentual de patentes por área de aplicação da tecnologia de acordo com a Classificação Internacional de Patentes (IPC)

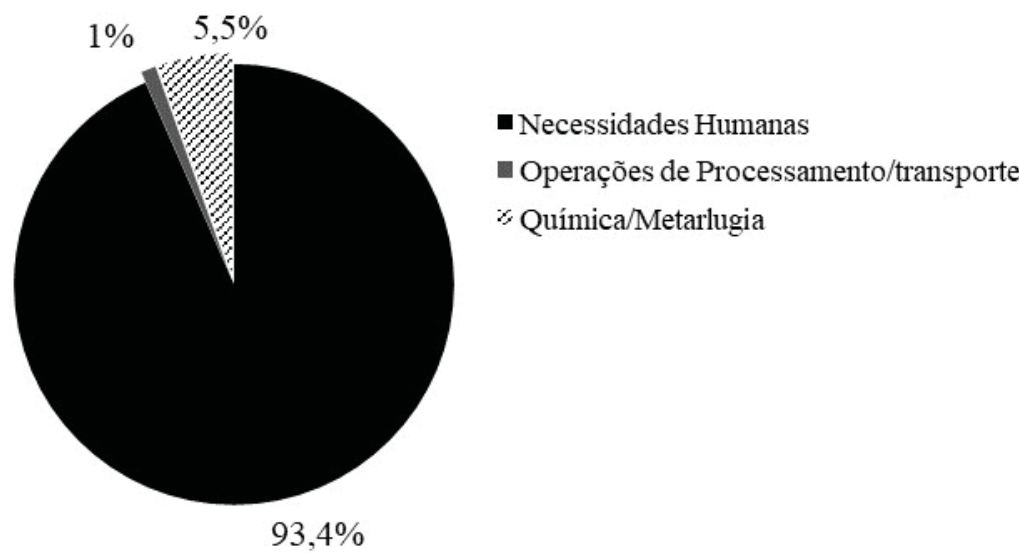

Fonte: Elaborado pelos autores deste artigo (2018)

Os códigos de identificação do IPC de maior frequência nas patentes relacionadas a $\mathrm{Ne}$ cessidades Humanas foram A23L (alimentos, produtos alimentícios ou bebidas não alcoólicas), seguida de A01D (Agricultura) e de A23K (produtos alimentícios e métodos de preparação, e os especialmente adaptados para animais) (Gráfico 4). Isso evidencia o aproveitamento tecnológico do Cyperus esculentus para o desenvolvimento de produtos com finalidades alimentícias, 
confirmando que a espécie detém um grande potencial para a indústria de alimentos. Essa tendência é atribuída a compostos que atribuem valor nutricional à planta, como carboidratos (OLADELE; AINA, 2007; ARAFAT et al., 2009), proteínas, lipídios e minerais (ADEGUNWA; ADELEKAN; ADEBOWALE, 2017). Nesse sentido, a principal aplicação tecnológica A23L corrobora com as publicações científicas referentes à importância nutricional, agregando valor econômico ao Cyperus esculentus.

Gráfico 4 - Frequência de códigos de identificação das patentes relacionadas ao Cyperus esculentus, de acordo a Classificação Internacional de Patentes (IPC)

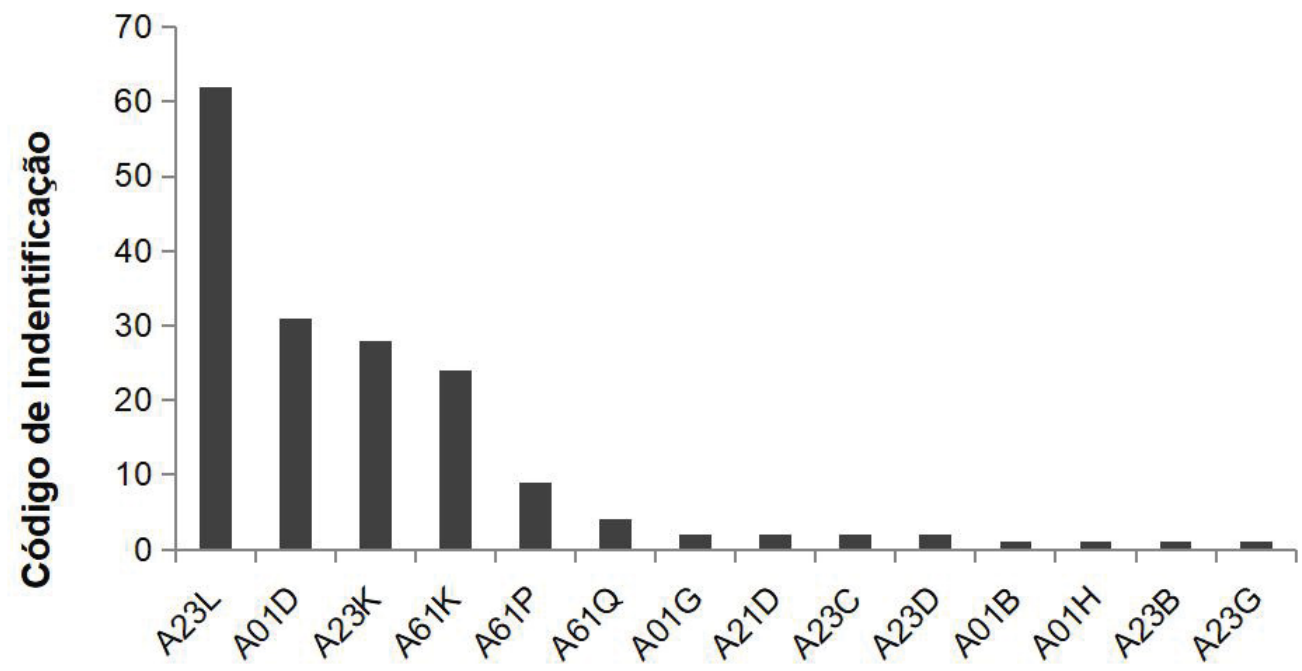

Classificação Internacional de Patentes

Fonte: Elaborado pelos autores deste artigo (2018)

Considerando-se a aplicação terapêutica da planta, foi observado o registro de cinco patentes relacionadas ao potencial farmacológico. Nesse contexto, a classificação A61K foi a mais frequente e trata de invenções com finalidades médicas, odontológicas ou higiênicas, com destaque para preparações farmacêuticas. Outro código que também aparece é o A61P, que é caracterizado por enquadrar atividades terapêuticas de compostos químicos e de preparações medicinais. As patentes encontradas com esses códigos visam à utilização da espécie para tratamento capilar, combate ao envelhecimento e estresse emocional, e efeitos antidiabéticos e antibacterianos contra Bacillus subtilis e Escherichia coli (Quadro 1), demonstrando a aplicação tecnológica de propriedades terapêuticas da planta. 
Quadro 1 - Lista de patentes relacionadas ao potencial farmacológico da espécie Cyperus esculentus

\begin{tabular}{|c|c|c|c|c|}
\hline $\begin{array}{l}\text { Número da } \\
\text { Patente }\end{array}$ & País & Ano & Título & IPC \\
\hline DE102012203989 & Alemanha & 2012 & $\begin{array}{l}\text { COMPOSITION, USEFUL TO TREAT HAIR, } \\
\text { COMPRISES CYPERUS ESCULENTUS } \\
\text { TUBER EXTRACT, A QUATERNARY } \\
\text { AMMONIUM COMPOUND E.G. AN } \\
\text { ESTERQUAT OR POLYQUATERNIUM-74, } \\
\text { AND AN AMINO FUNCTIONAL SILICONE }\end{array}$ & $\begin{array}{l}\mathrm{A} 61 \mathrm{~K} 8 / 41 \\
\mathrm{~A} 61 \mathrm{~K} 8 / 898 \\
\mathrm{~A} 61 \mathrm{~K} 8 / 97\end{array}$ \\
\hline US2010291250 & $\begin{array}{l}\text { Estados } \\
\text { Unidos }\end{array}$ & 2009 & $\begin{array}{c}\text { USE OF AN ACTIVE INGREDIENT THAT } \\
\text { IS OBTAINED FROM CYPERUS } \\
\text { ESCULENTUS FOR ITS ANTIAGING } \\
\text { CUTANEOUS ACTION }\end{array}$ & $\begin{array}{l}\text { A61K36/8905; } \\
\text { A61P43/00; } \\
\text { A61Q19/08; } \\
\text { A61K36/8905 }\end{array}$ \\
\hline CN104083571 & China & 2014 & $\begin{array}{l}\text { EXTRACTION METHOD AND USE OF CYPERUS } \\
\text { ESCULENTUS GENERAL FLAVONE CAPABLE } \\
\text { OF ENHANCING MICROCIRCULATION }\end{array}$ & $\begin{array}{l}\text { A61K36/8905 } \\
\text { A61P39/06 } \\
\text { A61P7/02 }\end{array}$ \\
\hline RU2197259 & Rússia & 2001 & ANTIDIABETIC AGENT & $\begin{array}{l}\text { A61K36/8905; } \\
\text { A61P3/00; } \\
\text { A61P3/10; } \\
\text { A61K125/00; } \\
\text { A61K35/78; } \\
\text { A61P3/00 }\end{array}$ \\
\hline RU2176516 & Rússia & 2000 & STRESS CORRECTING ADAPTOGEN & $\begin{array}{l}\text { A61K36/185 } \\
\text { A61P25/00 } \\
\text { A61P43/00 }\end{array}$ \\
\hline
\end{tabular}

Fonte: Elaborado pelos autores deste artigo (2018)

Em relação ao perfil dos depositantes, verificou-se que o percentual de depósitos por pessoa física (inventores independentes) é bem maior em relação à empresa e universidade, representando 63\% dos registros de patentes (Gráfico 5). Segundo Mueller e Perucchi (2014), vários países têm incentivado a inserção das universidades na produção de patentes, e os depósitos de patentes universitárias têm aumentado entre a década de 1990 e a primeira década do século XXI.

Gráfico 5 - Percentual dos documentos de patentes sobre a espécie Cyperus esculentus por tipo de depositante

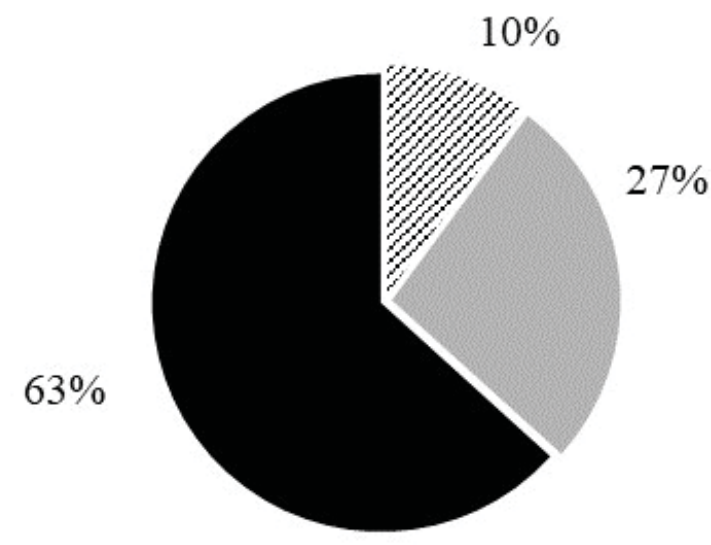

«niversidade

Empresa

- Pessoa Física

Fonte: Elaborado pelos autores deste artigo (2018) 
De acordo com a análise de distribuição de depósitos de patentes por país de origem da tecnologia, a China foi o país de maior destaque, responsável por $84 \%$ dos registros (Gráfico 6), sendo considerada uma das maiores potências em depósitos de patentes, com destaque em diferentes áreas, apresentado indicadores de investimento em pesquisa, desenvolvimento $e$ inovação (COSTA et al., 2018; MARQUES et al., 2019).

Gráfico 6 - Principais países detentores de patentes relacionadas ao C. esculentus

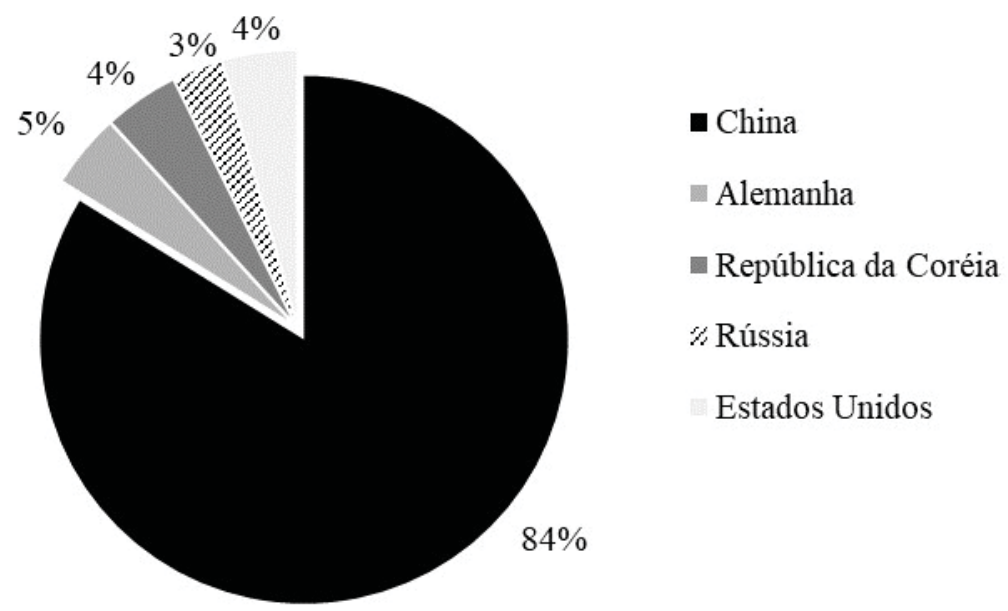

Fonte: Elaborado pelos autores deste artigo (2018)

O Brasil não aparece entre os países detentores de tecnologias com Cyperus esculentus, mesmo apresentando publicações científicas relacionadas à espécie. Em 2004, foi aprovada a Lei de Inovação, que busca facilitar a transferência de tecnologia, e um dos seus efeitos foi a criação de Núcleos de Inovação Tecnológica (NITs) nas universidades (MUELLER; PERUCCHI, 2014). Entretanto, o Brasil ainda possui baixo investimento na área de propriedade intelectual $e$, consequentemente, falta incentivo para proteger invenções no país, bem como para criar estratégias que articulem a academia e o setor empresarial (COSTA et al., 2015). A falta de comunicação entre as universidades brasileiras e a indústria tem se caracterizado como um dos grandes obstáculos para a geração de tecnologias (OLIVEIRA; NISHIJIMA, 2018).

\section{Considerações Finais}

As invenções tecnológicas referentes ao Cyperus esculentus demonstram a aplicação biotecnológica da espécie agregando importância científica, tecnológica e econômica, com destaque para publicações e patentes com finalidades alimentícias. Os resultados também evidenciam as potencialidades tecnológica e científica da espécie para a indústria farmacêutica, pois as publicações e patentes analisadas revelam a aplicação terapêutica da espécie, bem como a presença de compostos bioativos. Sendo assim, os documentos relacionados à Cyperus esculentus revelam o seu potencial para atender as necessidades humanas na área de alimento e saúde, podendo ser explorada pela indústria alimentícia, de cosmético e farmacêutica, com perspectiva para geração de novas tecnologias. 


\section{Referências}

ADEBOWALE, A. A.; KAREEM, S. T.; SOBUKOLA, O. P. Mineral and antinutrient contente of high quality cassava-tigernut compositive flour extruded Snack. Journal of Food Processing and Preservation, [S.I.], v. 41, 2017.

ADEGUNWA, M. O.; ADELEKAN, E. O.; ADEBOWALE, A. A. Evaluation of nutritional and functional properties of plantain (Musa paradisíaca L.) and tigernut (Cyperus esculentus L.) flour blends for food formulations. Congent Chemistry, [S.I.], v. 3, 2017.

ADEJUYITAN, A. Tigernut processing: its food uses and health benefits.American Journal of Food Technology, [S.l.], v. 6, p. 197-201, 2011.

ADENIYI, T. A.; ADEONIPEKUN, P. A.; OMOTAYO, E. A. Investigating the Phytochemicals and Antimicrobial Properties of Three Sedge (Cyperaceae) Species. Notulae Scientia Biologicae, [S.l.], v. 6, p. 276-281, 2014.

AGUILAR, N. et al. Chickpea and tigernut flours as alternatives to emulsifier and shortening in gluten-freebread. Food Science and Technology, [S.I.], v. 62, p. 225-232, 2015.

ADEWUYI, A.; ODERINDE, R. A.; AYODELE, G. A. Antimicrobial activity of diethanolamide: a nonionic surfactant from Calophyllum inophyllum and Cyperus esculentus. La Rivista Italiana Delle Sostanze Grasse, [S.l.], v. 89, p. 112-116, 2012.

ALLOUH, M. H.; DARADKA, H. M.; GHAIDA, H. J. H. A. Influence of Cyperus esculentus tubers (Tiger Nut) on male rat copulatory behavior. BMC Complementary and Alternative Medicine, [S.l.], v. 15, p. 331-335, 2015.

ARAFAT, S. M. et al. Chufa Tubers (Cyperus esculentus L.): As a New Source of Food. World Applied Sciences Journal, [S.1.], v. 7, p. 151-156, 2009.

ARIAS-PEREZ, F. H.; ESPINOSA-CAMILO, O. L.; DOMINGUEZ-MONGE, S. New host os Cyrtomenus crassus Walker in the Central Valley os Mexico. Southwestern Entomologist, [S.l.], v. 42, p. 601-604, 2017.

BADO, P. S. et al. Physicochemical characteristics and composition of three morphotypes of Cyperus esculentus tubers and tuberoils. Journal of Analytical Methods in Chemistry, [S.l.], v. 2.015, 2015.

CODINA-TORRELLA, I.; GUAMIS, B.; FERRAGUT, V. Potencial application of ultra-high pressure homogenization in the physico-chemical stabilization of tiger nuts'milk beverage. Innovative Food Science \&Emerging, [S.I.], v. 40, p. 42-51, 2017.

COSTA, T. O.; COSTA, L. E. C.; FREITAS, A. P. Mapeamento de patentes de formas farmacêuticas de desintegração oral. Revista INGI, [S.I.], v. 2, n. 2, p. 109-115, 2018.

COSTA, N. J. et al. Technological and therapeutic potential of Calotropis procera. Revista GEINTEC, [S.l.], v. 5, p. 2.222-2.236, 2015.

FOLLAK, S. et al. Biological flora of Central Europe: Cyperus esculentus L. Perspectives in Plant Ecology, Evolution and Sytematics, [S.I.], v. 23, p. 33-51, 2016.

GUSMAN-KANTUN, S.; ESPINOSA-CAMILO, O. L.; CAMPOS-FIGUEIROA, M. New report of Phenacoccus solani Ferrison Cyperus esculentus L. in the Valley of Mexico. Southwestern

Entomologist, [S.I.], v. 42, p. 305-308, 2017. 
HAMDY, S. M. et al. Protective effect of hesperidin and tiger nut against acylamide toxicity in female de rats. Experimental and Toxicologic Pathology, [S.I.], v. 69, p. 580-588, 2017.

HOLM, L. G. et al. The World's Worst Weeds. Florida: Krieger Publishing Company; Holt, J.S., Orcutt, D.R., 1991.

JING, S. et al. The in vitro and in vivo antioxidant properties of Cyperus esculentus oil from Xinjiang, China. Journal of the Science of Food and Agriculture, [S.l.], v. 93, p. 1.505-1.509, 2013.

JING, S.; WANG, S.; Dynamic high pressure microfluidization-assisted extraction and bioactivities of Cyperus esculentus (C. esculentus L.) leaves flavonoids. Food Chemistry, [S.l.], v. 192, p. 316-327, 2016.

JOHNSON, W. C.; WAY, T. R.; BEALE, D. G. An undergraduate student projectto improve mechanical control of perennial nutsedges with a peanut digger in organic crop production. Weed Thechnology, [S.I.], v. 29, p. 861-867, 2015.

KISSMAN, K. G. Plantas infestantes e nocivas. São Paulo: BASF, 1997.

LIMA, D. F. et al. Technological forecasting of jaborandi (Pilocarpus Microphyllus): economically important specie in the north and northeast of Brazil. Revista GEINTEC, [S.1.], v. 5, p. 1.626-1.638, 2015.

LORENZI, H. Plantas daninhas do Brasil: terrestres, aquáticas, parasitas e tóxicas. Nova Odessa, SP: Inst. Plantarum, 2008.

MATOS, F. J. A.; CAVALCANTI, F. S.; PARENTE, J. P. Estudo agronômico qualitativo e quantitativo de Cyperus esculentus $L$. (junça) - Uma fonte inexplorada de alimento energético. Revista de Ciências Agronômica, [S.1.], v. 39, p. 124-129, 2008.

MARQUES, L. G. A. et al. Technological innovations of the 3d printer applied to health inovações tecnológicas da impressora 3D aplicada à saúde. Revista GEINTEC, [S.l.], v. 9, p. 5.191-5.203, 2019.

MELLO, S. C. M. Fungos e seus metabolitos no controle de tiririca. Emprapa: Recursos Géneticos e Biotecnologia, 2003.

MEYERS, S.; SHANKLE, M. W. An evaluation of pre-emergence metam-potassium and s-metolachlor for yellow nutsedge (Cyperus esculentus) management in sweetpotato. Weed Technology, [S.l.], v. 31, p. 436-440, 2017.

MHLANGA, B. et al. Weed as affected by maize (Zeamays L.)- cover crop rotations in contrast in garables oils of Zimbabwe underconservation agriculture. Crop Protection, [S.l.], v. 81, p. 47-56, 2016.

MUELLER, Suzana Pinheiro Machado; PERUCCHI, Valmira. Universidades e a produção de patentes: tópicos de interesse para o estudioso da informação tecnológica. Perspectivas em Ciência da Informação, [S.l.], v. 19, p. 15-36, 2014.

OKYEREA, C. A. A.; ODAMTTENB, G. T. Physicochemical, functional and sensory attributes of milk prepared from irradiated tigernut (Cyperus esculentus L.) Journal of Radiation Research and Applied Sciences, [S.l.], v. 7, p. 583-588, 2014. 
OLADELE, A. K.; AINA, O. Chemical composition and functional properties of flour produced from two varieties of tigernut (Cyperus esculentus). African Journal of Biotechnology, [S.l.], v. 6, p. 2.473-2.476, 2007.

OLIVEIRA JR., R. S; CONSTANTIN, J.; INOUE, M. H. Biologia e Manejo de Plantas Daninhas, [S.l.]: Omnipax Editora, 2011.

OLIVEIRA, M. F.; NISHIJIMA, M. Os direitos de propriedade intelectual na indústria farmacêutica: análise comparativa da indústria brasileira com Índia e China. Revista de Economia Mackenzie, [S.l.], v. 15, 2018.

PRAKASH, N.; RAGAVAN, B. Phytochemical observation and antibacterial activity of Cyperus esculentus L. Ancient Science of Life, [S.I.], v. 28, p.16-20, 2009.

RUBERT, J.; MONFORTE, A.; HURKOVA, K. Untargeted metabolomics of fresh and heat treatment tiger nut (Cyperus esculentus L.) milks reeals further insight into food quality and nutrition. Journal of Chromatographya, [S.I.], v. 1.514, p. 80-87, 2017.

SAGANUWAN, A. Tropical plants with fertility and infertility value. Journal of the Europe Menopause and Andropause Society (EMAS), [S.1.], v. 18, p. 195-196, 2015.

SEUKEP, J. A. et al. Antibacterial activities of the methanol extracts of seven Cameroonian dietary plants against bacteria expressing MDR phenotypes. Springer Plus, [S.I.], v. 2, p. 2-8, 2013.

SIMÕES, E. R. B. et al. Technological Forecastingon Phytotherapics Development in Brazil. World Academy of Science, Engineering and Technology, [S.1.], v. 67, p. 132-136, 2012.

WANG, W. et al. Biodiesel production from hydrolysate of Cyperus esculentus waste by Chlorella vulgaris. Bioresource Technology, [S.I.], v. 136, p. 24-29, 2013.

\section{Sobre os Autores}

\section{Renata Correia}

E-mail: renata.correia@uesb.edu.br

Doutorado em Biotecnologia (Recursos Naturais) pela Universidade Federal da Bahia (2018).

Endereço profissional: Universidade Estadual do Sudoeste da Bahia, Estrada do Bem Querer, s/n, Bairro Universitário, Vitória da Conquista, BA. CEP: 45000-000.

\section{Reinaldo Assunção Júnior}

E-mail: reinaldojr92@gmail.com

Especialização em Gestão da Cadeia Produtiva do Café com Ênfase em Sustentabilidade pela Universidade Estadual do Sudoeste da Bahia (2018).

Endereço profissional: Rua Uberlândia, s/n, Ipanema, MG. CEP: 39990-000.

\section{Romulo Spósito}

E-mail: rsvagro@gmail.com

Mestrado em Zootecnia (produção de Ruminantes) pela Universidade Estadual do Sudoeste da Bahia (2012).

Endereço profissional: Instituto Federal de Educação, Ciência e Tecnologia Baiano, Campus Itapetinga, BA,

Rodovia Itapetinga-Itororó Km 2, Clerolândia, Itapetinga, BA. CEP: 45700-000. 


\section{Cristina Pungartnik}

E-mail: cpungartnik@gmail.com

Doutorado em Ciências Biológicas (Bioquímica) pela Universidade Federal do Rio Grande do Sul (1999).

Endereço profissional: Universidade Estadual de Santa Cruz, Rodovia Ilhéus, Itabuna Km 16, s/n, Ilhéus, BA. CEP: 45662-000. 